Check for updates

Cite this: Phys. Chem. Chem. Phys., 2019, 21, 10961

Received 12th December 2018 Accepted 26th March 2019

DOI: $10.1039 / c 8 c p 07592 a$

rsc.li/pccp

\title{
Relative stability of diamond and graphite as seen through bonds and hybridizations
}

\author{
Ilya V. Popov, ${ }^{\text {ab }}$ Arno L. Görne, ${ }^{c}$ Andrei L. Tchougréeff (D) *abc and \\ Richard Dronskowski (D)*cd
}

\begin{abstract}
The relative stability of the two most important forms of elemental carbon, diamond and graphite, is readdressed from a newly developed perspective as derived from historically well-known roots. Unlike other theoretical studies mostly relying on numerical methods, we consider an analytical model to gain fundamental insight into the reasons for the quasi-degeneracy of diamond and graphite despite their extremely different covalent bonding patterns. We derive the allotropes' relative energies and provide a qualitative picture predicting a quasi-degenerate electronic ground state for graphite (graphene) and diamond at zero temperature. Our approach also gives numerical estimates of the energy difference and interatomic separations in good agreement with experimental data and recent results of hybrid DFT modeling, although obtained with a much smaller numerical but highly transparent effort. An attempt to extend this treatment to the lowest energy allotropes of silicon proves to be successful as well.
\end{abstract}

\section{Introduction}

Carbon allotropes are a hot topic with a plethora of newly proposed ones, predicted to have fascinating physical properties. To navigate this large zoo of allotropes, we recommend a comprehensive and timely database, ${ }^{1}$ a survey ${ }^{2}$ and two recent articles dealing with the bonding of these carbon allotropes ${ }^{3}$ and sp-mixing triangle maps from first principles. ${ }^{4}$ The experimentally available allotropes are by far less numerous but of interest in different areas: graphene and fullerenes are popular materials in material sciences and electrochemistry, ${ }^{5,6}$ and modified nanotubes have been found to be efficient catalytic systems. ${ }^{7}$ Despite the impressive diversity of novel carbons, the well-known diamond and graphite (Fig. 1, as well as hexagonal diamond dubbed lonsdaleite) form the centerpiece of the entire carbon story. These two allotropes have been known to humans for thousands of years, being the only crystalline forms present in nature rather than prepared artificially. Also, they are the most studied carbons and prototypical for other allotropes variously combining $\mathrm{sp}^{3}$ - and $\mathrm{sp}^{2}$-hybridized $\mathrm{C}$ atoms. ${ }^{2,11-13}$ Remarkably, diamond itself can exist in two forms, the usual cubic diamond and hexagonal lonsdaleite ${ }^{10}$ featuring the same

\footnotetext{
${ }^{a}$ A.N. Frumkin Institute of Physical Chemistry and Electrochemistry of the Russian Academy of Science, Moscow, Russia

${ }^{b}$ Independent University of Moscow, Moscow, Russia

${ }^{c}$ Chair of Solid State and Quantum Chemistry, RWTH Aachen University,

52056 Aachen, Germany.E-mail: andrei.tchougreeff@ac.rwth-aachen.de, drons@HAL9000.ac.rwth-aachen.de

${ }^{d}$ Jülich-Aachen Research Alliance, JARA-HPC, RWTH Aachen University, 52056 Aachen, Germany
}
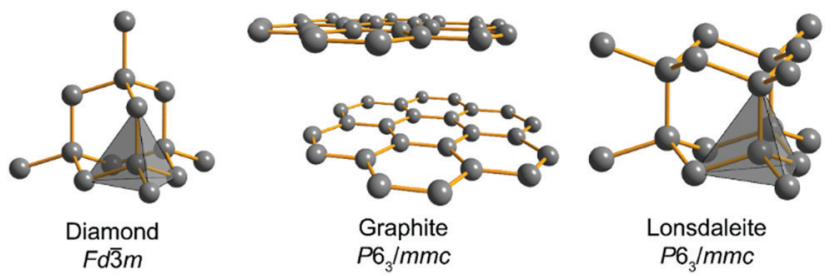

Fig. 1 Crystal structures of diamond, ${ }^{8}$ graphite $^{9}$ and lonsdaleite, the hexagonal form of diamond. ${ }^{10}$

local arrangements of $\mathrm{C}-\mathrm{C}$ bonds but differing by the hexagonal $\mathrm{ABAB}$ stacking as compared to the cubic ABCABC one (Fig. 1).

The most intriguing fact about diamond and graphite is their relative stability. Experimentally, graphite is thermodynamically more stable at $1 \mathrm{~atm}$ and $298 \mathrm{~K}$, but only barely $\left(c a .2 \mathrm{~kJ} \mathrm{~mol}^{-1}\right.$, that is, they are degenerate within a chemical accuracy of $1 \mathrm{kcal} \mathrm{mol}^{-1}$ ). Any attempt to find a rigorous explanation of this quasi-degeneracy stumbles upon the problem that diamond and graphite are just too different from the point of view of, say, topology, which is accepted as a basis of allotrope classification in ref. 1. Indeed, diamond and graphite adopt different space-group symmetry, different unit cells, different band structures and extremely different physical properties, so why should they have (almost) equal energies? We recall that solid-state chemistry would portray diamond and graphite as species featuring different hybridization patterns $\left(\mathrm{sp}^{3} v s\right.$. $\left.\mathrm{sp}^{2}\right)$, different localization, and different covalent bonding types. Thus, it might look easier to explain if graphite were significantly more stable since the delocalized $\pi$-system might also decrease graphite's total 
energy as habitually evoked in organic chemistry. Vice versa, if diamond were significantly more stable, one might argue in terms of the larger number of strong covalent bonds per atom. A recent, comprehensive DFT study of the relative stability of diamond and graphite ${ }^{14}$ provides valuable numerical information on the exact allotropic energy difference under different conditions, but in our contribution, we try to understand how diamond and graphite can come so close on the energy scale in terms of an analytical model.

\section{Experimental situation}

First, we specify the quantity to be reproduced. Under standard conditions, the difference in Gibbs energies $\Delta G^{\circ}$ between diamond and graphite falls in the range of $2.87-2.90 \mathrm{~kJ} \mathrm{~mol}^{-1}$, graphite being lower, ${ }^{15}$ but this quantity is contaminated by the lattice contribution. Since the crystal structures and elasticity modules and, thus, the phonon spectra are rather different, one cannot a priori exclude changes in the order of electronic energies. We therefore extrapolated the difference in Gibbs energies to zero temperature; the standard experimental data (NIST) of entropy and heat capacities for graphite (see Appendix A) ${ }^{16}$ are fit to the Debye-Sommerfeld model, $C_{\mathrm{p}}=\gamma T+\alpha T^{3}$. For diamond, this yields $\gamma=0$ (nonmetallic and no other degrees of freedom yielding a linear term) but graphite features so called flexural vibrations (Fig. 2), ${ }^{17}$ whose dispersion law produces a $T^{2}$ contribution to the low-temperature heat capacity, as predicted quite long ago ${ }^{19}$ and documented experimentally. ${ }^{20}$ Even with accounting for the flexural modes, graphite is still more stable than diamond at $0 \mathrm{~K}$, but the difference in Gibbs energies is even smaller (1.39-1.55 $\mathrm{kJ} \mathrm{mol}^{-1}$ ) than at room temperature. Taking into account the ZPEs (as estimated in ref. 14) of either allotrope yields a difference of electronic energies in the range of $0.14-0.78 \mathrm{~kJ} \mathrm{~mol}^{-1}$ in favor of graphite.

Another uncertainty is the weak interaction between the graphene layers forming graphite. To avoid this overwhelmingly complex problem (in particular when relying on DFT approaches), we notice that adding the recently ${ }^{21}$ determined exfoliation energy of graphite (about $3 \mathrm{~kJ}$ per mole of carbon atoms) puts the electronic energy of an individual graphene sheet $c a .2 .5-3 \mathrm{~kJ} \mathrm{~mol}^{-1}$

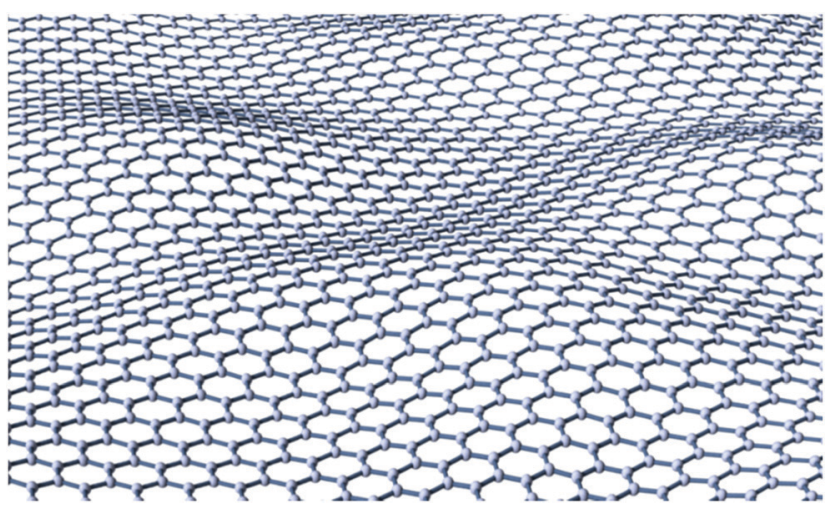

Fig. 2 A snapshot of a flexural vibration of an individual graphite sheet yielding the $T^{2}$ contribution to the heat capacity. ${ }^{18}$ above diamond. This suggests that, within chemical accuracy, diamond and graphene (and graphite, too) are almost degenerate states of carbon. Thus, we use graphene in our considerations for reasons of simplicity.

\section{Model}

Now, we turn to constructing an analytical model of the electronic structures for both carbon allotropes, relying upon fundamental chemical concepts such as bonding, hybridization etc. Like in the Molecular Mechanics or Force Field ${ }^{22-27}$ approaches, we represent the energy of each allotrope as a sum of energies of the bonds formed by differently hybridized orbitals, complemented by the energies of atoms in the respective hybridization states and by the energy of repulsion of the atomic "cores", that is, the atoms bereft of their valence electrons. $\dagger$ Instead of a priori inferring the form of the bond energies, we use expressions stemming from a general quantum-mechanical theory of the molecular electronic structure, the formal background being the group-function formalism. ${ }^{28-30}$ The diamond energy per atom is that of the $\mathrm{sp}^{3}$ hybridized carbon atom supplied by the energies of two $\sigma$-bonds formed by these hybrids (four $\sigma$-bonds counted $50 \%$ per atom) and the structure-dependent repulsion energy. Likewise, graphene contains $3 / 2 \sigma$-bonds formed by $\mathrm{sp}^{2}$ hybrids per atom, but their energy must be augmented by that of a delocalized $\pi$-electron per atom. Thus, we write

$$
\begin{aligned}
E_{\text {Diamond }} & =E_{0}\left(\mathrm{sp}^{3}\right)+2 E_{\sigma}\left(\mathrm{sp}^{3}\right)+E_{\text {rep }}^{(\mathrm{D})} \\
E_{\text {Graphite }} & =E_{0}\left(\mathrm{sp}^{2}\right)+\frac{3}{2} E_{\sigma}\left(\mathrm{sp}^{2}\right)+\frac{3}{2} E_{\pi}+E_{\text {rep }}^{(\mathrm{G})}
\end{aligned}
$$

and call $E_{0}(h)$ the hybridization-dependent one-center energies, that is, the sums of core attraction and mutual repulsion of the valence electrons in the atoms. $E_{\sigma}(h)$ are the energies of $\sigma$ bonds formed by the $\mathrm{sp}^{3}$ and $\mathrm{sp}^{2}$ hybrid orbitals, $E_{\pi}$ is the energy of the $\pi$-electrons in graphene recalculated per number of nearest neighbors of each atom, so the multipliers equal 3/2, and $E_{\text {rep }}^{(\mathrm{A})}$ represents the core-core repulsions of the carbon atoms specific for each allotrope due to structural differences.

Clearly, the carbon atoms in the allotropes have different hybridizations. Normally, the one-center energies $E_{0}(h)$ strongly depend on hybridization, ${ }^{31-33}$ but the allotropes of the group IV elements represent a remarkable exception. ${ }^{31}$ For them, the populations of all atomic hybrid orbitals are equal to unity, and the one-center terms are equal in graphene and diamond (see proof in Appendix B), so we can exclude them from now on.

The repulsion energies $E_{\text {rep }}^{(\mathrm{D})}$ and $E_{\text {rep }}^{(\mathrm{G})}$ come from the general formula for the repulsion of the atomic charge distribution if one takes into account the differences between interactions of the core point charges and of the electron distributions with a spatial extent. ${ }^{30,32}$ Hence, repulsive interactions between electroneutral atoms acquire a quasi-Yukawa form $Y(d)$, that is, $d^{-1}$

$\dagger$ For the carbon atom, the effective core charge arrives at +4 because there is an electronic configuration of $1 \mathrm{~s}^{2} 2 \mathrm{~s}^{2} 2 \mathrm{p}^{2}$ from which the $2 \mathrm{~s}^{2} 2 \mathrm{p}^{2}$ valence electrons are separated, leaving the core containing the +6 atomic nucleus plus the $1 \mathrm{~s}^{2}$ electrons together charged as +4 . 
multiplied by exponentials assuring a faster decay at longer distances $d$, and we may write

$$
E_{\text {rep }}^{(\mathrm{A})}=\frac{Z^{2}}{2} \sum_{b \neq a} Y\left(d_{a b}\right),
$$

where $Z$ is the core charge ( +4 for carbon, see above) and the summation over $b$ extends to all other atoms in the crystal. The nearest-neighbor ( $\mathrm{nn}$ ) core repulsion therefore hugely prefers graphene over diamond, $E_{\mathrm{rep}, \mathrm{nn}}^{(\mathrm{G})} E_{\mathrm{rep}, \mathrm{nn}}^{(\mathrm{D})}=3 / 4$ for equal interatomic separations, so diamond's higher coordination number makes it energetically less favorable. ${ }^{34}$

In quantum chemistry, the bond energies may be expressed through quantum-mechanical quantities such as the Coulson bond order $P .{ }^{35}$ For the energy of the bonding interactions between two equivalent orbitals of hybridization $h$, one has

$$
E(h)=-\frac{1}{2}\left(4 \beta_{\mathrm{h}} P_{\mathrm{h}}+g_{\mathrm{nn}} P_{\mathrm{h}}^{2}\right),
$$

where $\beta_{\mathrm{h}}$ is the one-electron hopping matrix element between the hybridized orbitals, $P_{\mathrm{h}}$ is the Coulson bond order of hybridization $h$ and $g_{n n}$ is the Coulomb interaction energy between electrons residing in the involved orbitals. Because of the transferability theorem, ${ }^{36-38}$ the Coulson bond orders are almost geometry- and composition-independent such that, for two-center two-electron carbon-carbon single bonds formed by $\mathrm{sp}^{2}$ or $\mathrm{sp}^{3}$ hybrids, ${ }^{39} P_{\sigma}^{(\mathrm{D})} \approx P_{\sigma}^{(\mathrm{G})} \approx 1$.

In the delocalized $\pi$-system of graphene, the nearest-neighbor Coulson bond order derives from the band (equivalent to Hückel) approximation. Its solution for the graphene $\pi$-system ${ }^{40}$ yields $P_{\pi} \approx 0.525$ (see, for example, ref. 59). Just like for the $\sigma$-bonds, it is parameter- and distance-independent, defined exclusively by the "topology" of the graphene lattice. Since the graphene lattice divides into two sublattices, so that sites of one sublattice have neighbors only in another one, the alternation theorem ${ }^{41}$ holds for graphene's $\pi$-bond orders. According to this, all bond orders between orbitals from the same sublattice - that is, the secondnearest ones - vanish. Therefore, despite the delocalization, we may retain only the nearest-neighbor interactions in the $\pi$-system. Further neighbors fall out due to the exponential decay of the hopping (resonance) interactions.

That being said, the energies of diamond and graphene are just two different functions of the interatomic separation, comprising Coulomb repulsion $g_{\text {nn }}$, core-core repulsion $Y$, and orbital-orbital hopping elements $\beta_{\mu \nu}$ for which all quantum-mechanical electronic-structure variables (hybridizations and bond orders) are fixed by the symmetry. The only remaining variable, the interatomic separation $d$, enters through the exponentially decaying terms

$$
E_{\mathrm{G}, \mathrm{D}}(d) \asymp-B_{\mathrm{G}, \mathrm{D}} \exp (-\xi d)+D_{\mathrm{G}, \mathrm{D}} \exp (-\alpha d)
$$

where “こ” stands for asymptotically equal, producing a kind of "generalized" Morse potential. $\$^{42}$ The difference of the allotropes' energies as a function of $d$ has a similar form

$$
\Delta_{\mathrm{DG}} E(d) \asymp-\Delta_{\mathrm{DG}} B \exp (-\xi d)+\Delta_{\mathrm{DG}} D \exp (-\alpha d)
$$

\$ We remind the reader that in the true Morse potential, we have $\alpha=2 \xi$. namely, two terms with the same exponents supplied by preexponential factors. Setting this to zero defines an intermediate separation or degeneracy point $d_{*}$ for which the energies of diamond and graphene are equal:

$$
d_{*}=\frac{\ln \Delta_{\mathrm{DG}} D-\ln \Delta_{\mathrm{DG}} B}{\alpha-\xi}
$$

This result is remarkable since it shows that the (quasi-)degeneracy of diamond and graphene is not accidental, but rather predetermined by the structure of the respective energy functions. Near the degeneracy point, the energies of the two allotropes expand up to the second power in $\left(d-d_{*}\right)$, as shown below. The energy difference between them is that of the stabilization energies at their respective equilibrium bond lengths, $d_{\mathrm{G}}<d_{*}<d_{\mathrm{D}}$ :

$$
\begin{aligned}
& E_{\mathrm{G}}(d) \approx E\left(d_{*}\right)+F_{\mathrm{G}}\left(d-d_{*}\right)+\frac{K_{\mathrm{G}}}{2}\left(d-d_{*}\right)^{2} \\
& E_{\mathrm{D}}(d) \approx E\left(d_{*}\right)+F_{\mathrm{D}}\left(d-d_{*}\right)+\frac{K_{\mathrm{D}}}{2}\left(d-d_{*}\right)^{2} \\
& \Delta_{\mathrm{DG}} E=\frac{F_{\mathrm{G}}^{2}}{2 K_{\mathrm{G}}}-\frac{F_{\mathrm{D}}^{2}}{2 K_{\mathrm{D}}}
\end{aligned}
$$

where $F_{\mathrm{G}, \mathrm{D}}$ and $K_{\mathrm{G}, \mathrm{D}}$ are the first (force) and second (rigidity) derivatives of the graphene and diamond energies at $d_{*}$.

\section{Implementation and results}

To proceed further, we need to specify the resonance integrals $\beta_{\mu \nu}(\mu, \nu=\mathrm{s}, \mathrm{p}, \pi)$ and the quasi-Yukawa potential $Y$. For the former, we extracted their distance dependence from densityfunctional calculations on graphite as projected on local atomic orbitals with the LOBSTER package ${ }^{43-45}$ (see Appendix C). The results of this extraction fit fairly well to the traditional semiempirical (MNDO) form of the resonance integrals $\beta_{\mu \nu}=\beta_{\mu \nu}^{0} S_{\mu \nu}$, where $S_{\mu \nu}$ are the overlap integrals of the Slater orbitals $\mu \nu$, and $\beta_{\mu \nu}^{0}$ are the scaling factors specific for the orbital type (s, p, $\pi$ ) and the chemical element. ${ }^{48}$ In view of the good agreement of the form of $\beta_{\mu \nu}$, it is reasonable to take them, as well as the quasi-Yukawa core repulsion $Y$, as given in the MNDO-based method $^{46,47}$ using the local bonds formed by the strictly local hybrids. This setting agrees with the asymptotic form of either allotrope energy (eqn (4)), assuring the existence of a degeneracy point.

The bonding energy contributions are depicted in Fig. 3a. Their slopes (= forces) are almost equal at the degeneracy point of $d_{*}=1.490 \AA$, differing by less than $3 \%$. Interestingly, the attractive force in graphene is smaller than in diamond as is the graphene attraction energy. The total force is the sum of the attractive and repulsive contributions partially compensating each other. For graphene, the repulsive force is always weaker than for diamond (Fig. 3b). At the degeneracy point, the total force is attractive for graphene (contracting the bond relative to $d_{*}$ ), but repulsive for diamond (stretching the bond relative to $d_{*}$ ). This ultimately leads to the energy profiles for carbon in the diamond and graphene structures shown in 

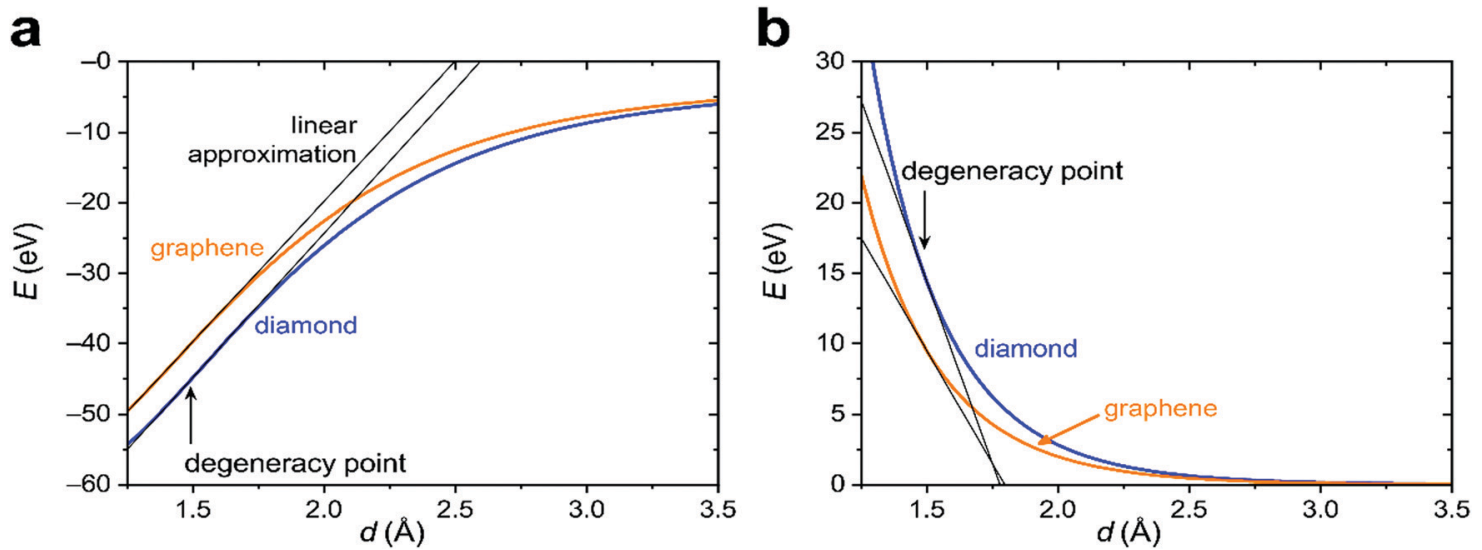

Fig. 3 Energy contributions of diamond and graphene structures of carbon as a function of the interatomic distances: (a) bonding energies of allotropes, (b) core-core repulsion. In (a) and (b), the linear approximation around the degeneracy point has been included (black lines).

Fig. 4a, which qualitatively agree with the experiments. More numerical results are given in Table 1.

According to our analysis, the observed relative energies of the allotropes (eqn (7)) appear due to a paradoxical interplay of factors: the weaker attractive force in diamond is compensated by a stronger repulsive force, producing a total repulsive force larger by its absolute value than the total attractive force in graphene. The difference in favor of the repulsive force is so large that when squared (numerator in the second term of the third row of eqn (7)), it still produces a stronger stabilizing effect despite the larger rigidity (corresponding denominator) of diamond. Comparing this to lonsdaleite, we find a slightly larger repulsive contribution of the 3rd and farther neighbors in the latter, which leads to a slightly larger energy of this allotrope and to a minor increase of the equilibrium interatomic separations as compared to cubic diamond.

One of the key elements leading to the energetic quasidegeneracy of the graphene and diamond structures in the carbon case is the independence of the atomic energy on hybridization, as shown in Appendix B. The reasoning given there applies to all group IV atoms. Thus, we also looked at the heavier homologue, silicon. Like for carbon, we first performed a DFT calculation on the Si crystal and extracted the resonance integrals (Appendix C), which also showed fair proportionality to the overlap integrals as expressed within MNDO theory. By using these parameters, the components of the attractive and repulsive forces as well as the rigidities were calculated for silicon at the degeneracy point, which is $d_{*}=1.977 \AA$. We then find that the bonding forces in silicon are smaller than in carbon by an order of magnitude, and the trend for silicon is opposite to carbon: the diamond structure has a smaller bonding force as compared to graphene (4.12 vs. $6.47 \mathrm{eV} \AA^{-1}$ ). Attractive forces are overcome by the repulsive ones, so that the overall forces in the case of either silicon allotrope turn out to be repulsive at the degeneracy point. For this very reason, the degeneracy point corresponds to a smaller interatomic separation than either of the equilibrium ones for silicon, unlike for the carbon case. Finally, the larger repulsive force characteristic of the diamond structure results in a stronger stabilization of the diamond structure (Table 1 and Fig. $4 \mathrm{~b}$ ) and sheds new light on the unavailability of silicene (silicon in the graphene structure).

Remarkably, the negative sign of the force acting on silicene at the degeneracy point corroborates with its instability towards
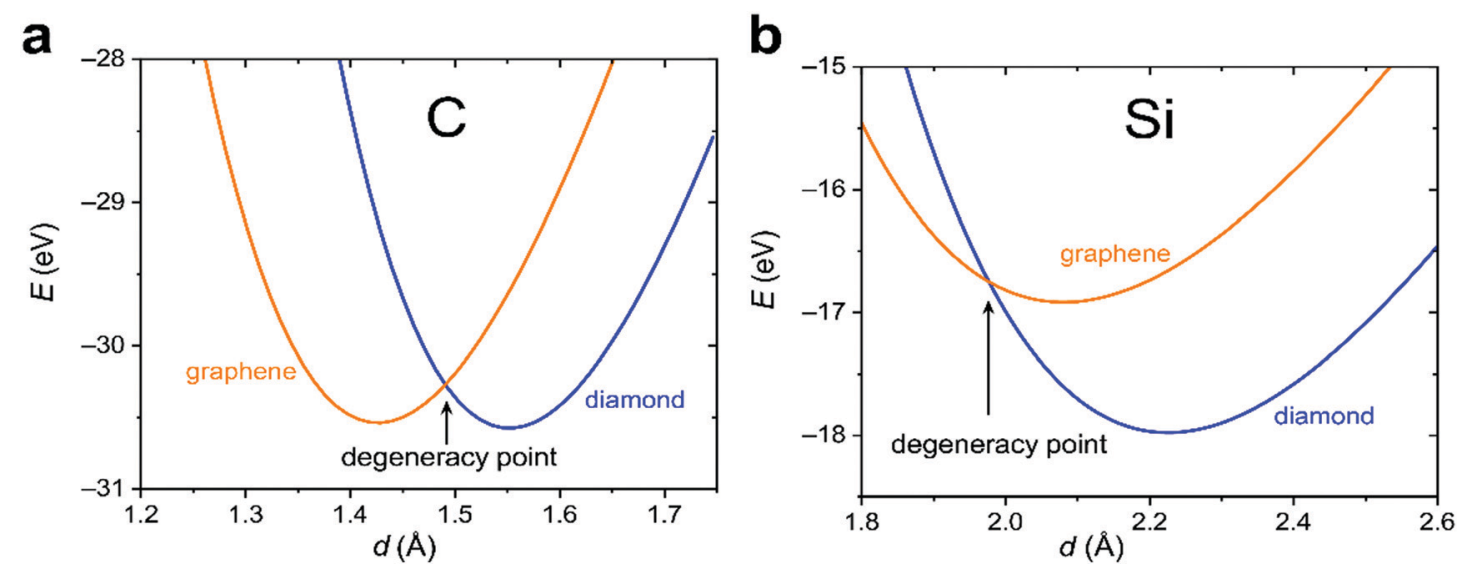

Fig. 4 Total-energy profiles (sums of the contributions from Fig. 3a and b) near the "degeneracy point" for the graphene and diamond structures of carbon (a) and silicon (b). 
Table 1 Calculated relative energies and interatomic separations in groupIV allotropes compared to the experimental values (where available). The calculated quantities are obtained using MNDO-like parameterization. ${ }^{47}$ $D, G$, and $L$ stand for the diamond, graphene and lonsdaleite ${ }^{d}$ structures

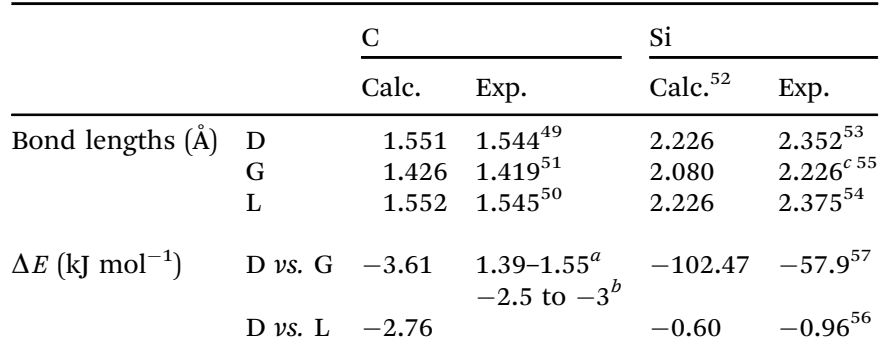

${ }^{a}$ This value is obtained in the present work (Appendix A) from the recommended value of $\Delta_{\mathrm{DG}} G^{\circ}$ and experimental data for the heat capacities. $^{16 b}$ The same including the exfoliation energy ${ }^{21}$ and the ZPE corrections. ${ }^{14 c}$ result of a DFT calculation. ${ }^{55 d}$ Lonsdaleite is the closest analogue of usual diamond featuring the same local environment, differing either by stacking (see the Introduction) or alternatively formulated by the conformation taken by the atoms adjacent to a given bond: eclipsed (lonsdaleite) vs. staggered (diamond). Although, there are some doubts ${ }^{70}$ as to whether lonsdaleite indeed exists in nature (at least in meteoritic craters, as originally conjectured $\left.{ }^{10}\right)$, for the purpose of the present paper, it is enough that the corresponding structure appears as a local energy minimum from a reasonable solid-state calculation, e.g. ref. 71 and 72 .

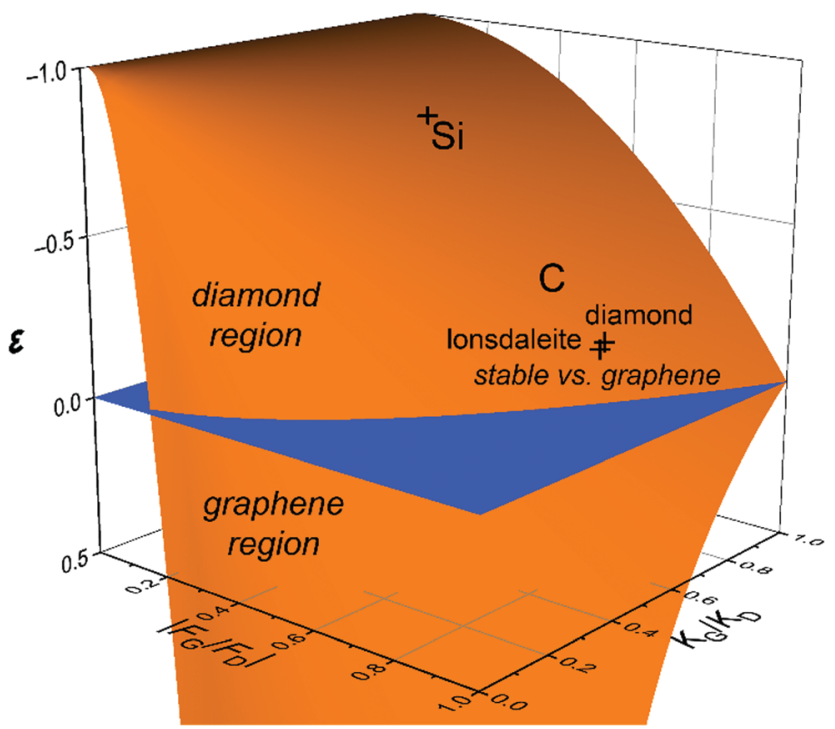

Fig. 5 General view of the relative energetics of the diamond and graphene allotropes of carbon in which the orange surface shows the ratio of the stabilization energies of the two allotropes relative to the degeneracy point shifted by unity: $\varepsilon=\left(F_{G}{ }^{2} / K_{G}\right):\left(F_{D}{ }^{2} / K_{D}\right)-1$. The axes are the ratios $\left|F_{\mathrm{G}} / F_{\mathrm{D}}\right|$ and $K_{\mathrm{G}} / K_{\mathrm{D}}$, the relative forces and rigidities for diamond and graphene at the degeneracy point.

corrugation. ${ }^{55}$ Indeed, given constant hybridization, the only effect of small corrugation is the replacement of the nearestneighbor interatomic separations $d$ by $d / \cos \alpha$ in all distancedependent functions. $\S$ For small values of $\alpha$, it produces the

$\S$ The corrugation angle $\alpha$ is the one between the graphene $\mathrm{C}-\mathrm{C}$ bond and the graphene plane. energy correction $F_{\mathrm{G}} \alpha^{2} / 2$, which for negative $F_{\mathrm{G}}$ (silicene), leads to an immediate instability of the planar structure, whereas in the case of carbon, the positive sign of $F_{\mathrm{G}}$ guarantees that the planar structure remains stable with respect to corrugation.

Another way of plotting the astonishing difference in energetics of the carbon and silicon allotropes is presented in Fig. 5, in which the horizontal axes are given by the relative forces and rigidities for diamond and graphene at the degeneracy point. In the blue plane, the intersecting line corresponds to the phase diagram with the above coordinates. Above the blue plane, diamond is preferred; below the blue plane, graphene is preferred. The energies of diamond and lonsdaleite relative to graphene are shown by crosses. Similarly, the energies of the diamond and lonsdaleite structures of Si relative to its graphene form (silicene) are shown; they coincide on this scale.

\section{Conclusions}

To summarize, we have performed a theoretical analysis of the relative stabilities of the lowest-energy carbon allotropes occurring in nature: graphite (graphene), diamond and lonsdaleite. Within our approach, we first excluded large and potentially imprecise contributions and then established the existence of an analytically given degeneracy point: the interatomic separation at which the energies of the allotropes coincide despite the drastic differences in their topology, spatial and electronic structures. This allowed us to shift the focus to the quantity of interest, that is, the difference of the allotropes' energies. In this setting, the sought energy difference comprises about $10 \%$ of the corrections to the energy at the degeneracy point.

Admittedly, one might think that the significant simplifications make our approach more qualitative than quantitative and do not permit us to arrive at an exact estimate of the relative energy of the lowest-energy allotropes of carbon and silicon. Quite to the contrary, it is puzzling to note the rather correct relative positioning on the energy scale almost arriving at "chemical accuracy" accessed by very simple means. Similarly, rather good numerical data for the interatomic separations (with an error smaller than $0.5 \%$ for carbon), comparable to high-level DFT calculations, $\uparrow$ have been achieved. All this was reached by making use of the empirical information about the general construction of the electronic wave functions of the respective allotropes (that is, form and character of the local and delocalized bonds) and a far smaller numerical effort than in hard-core numerical approaches (see, for example, ref. 13, 15 and 57). Clearly, the use of the bonding paradigm is important because, as mentioned, ${ }^{58}$ it allows one to keep track of chemical similarities not easily accessible otherwise. At the same time, the almost precise compensation of counteracting factors (that is, different hybridization, bonding pattern, and as well global and local structure) could also be reproduced in the case of graphene/ diamond if based on bonds and hybridizations.

T Even more, our numerical result agrees with the most recent coupled cluster calculation, ${ }^{73}$ also positioning diamond below graphite on the energy scale. 
We hope that our qualitative analysis might be helpful, providing a refreshing view on the carbon allotropy, complementary to other numerical and experimental approaches.

\section{Conflicts of interest}

There are no conflicts to declare.

\section{Theoretical appendix}

A. Overview of experimental data on the relative energies of allotropes. Which one is more stable at zero temperature?

The difference between the zero-point enthalpies of two ideal crystalline allotropes is given as

$$
\Delta_{\mathrm{DG}} H_{0}^{0}=\Delta_{\mathrm{DG}} G_{T}^{0}+T \Delta_{\mathrm{DG}} S_{T}^{0}-\int_{0}^{T} \Delta_{\mathrm{DG}} C_{\mathrm{p}} \mathrm{d} T
$$

where $\Delta_{\mathrm{DG}} G_{\mathrm{T}}^{0}$ is the difference between standard Gibbs free energies of diamond and graphite at temperature $T, \Delta_{\mathrm{DG}} S_{\mathrm{T}}^{0}$ is the difference between entropies of allotropes at temperature $T$ and $\Delta_{\mathrm{DG}} C_{\mathrm{p}}$ is the difference between constant-pressure heat capacities of the allotropes.

By substituting the experimental relative standard Gibbs energy and entropy at $300 \mathrm{~K},{ }^{60}$ we get

$$
\Delta_{\mathrm{DG}} H_{0}^{0}=1.878-\int_{0}^{300} \Delta_{\mathrm{DG}} C_{\mathrm{p}} \mathrm{d} T \mathrm{~kJ} \mathrm{~mol}^{-1}
$$

To arrive at the zero-temperature relative energy, the integral needs to be evaluated. This was done ${ }^{16}$ using experimental heat capacities of diamond and graphite, extrapolated to the zero temperature by the Debye-Sommerfeld relation: $C_{\mathrm{p}}=\gamma T+\alpha T^{3}$. The difference of zero temperature enthalpies $\Delta_{\mathrm{DG}} H_{0}^{0}$ obtained by this procedure amounts to $1.39-1.42 \mathrm{~kJ} \mathrm{~mol}^{-1}$.

In graphite, one must also take into account flexural vibrational modes (Fig. 2), yielding a quadratic term in the heat capacity. ${ }^{19,20}$ Thus, the formula $C_{\mathrm{p}}=\beta T^{2}+\alpha T^{3}$ must be used to extrapolate the heat capacity to zero temperature. Several sets of experimental points, recommended by NIST, ${ }^{61}$ were handled within this setting, and we found that the temperature correction to the enthalpy (sum of the entropic and the integral terms in eqn (A1)) of graphite lies in the range of 1.15-1.20 $\mathrm{kJ} \mathrm{mol}^{-1}$, which yields $\Delta_{\mathrm{DG}} H_{0}^{0}=1.48-1.55 \mathrm{~kJ} \mathrm{~mol}^{-1}$ for the difference of the zero-temperature enthalpies. Thus, the value of the zerotemperature enthalpy difference obtained, taking into account the flexural modes in graphite, is slightly larger than that evaluated from the data of ref. 16. However, the difference is insignificant $\left(0.1 \mathrm{~kJ} \mathrm{~mol}^{-1}\right)$. Thus, it is reasonable to expect that $\Delta_{\mathrm{DG}} H_{0}^{0}$ lies in the range of $1.39-1.55 \mathrm{~kJ} \mathrm{~mol}^{-1}$. Finally, the difference of ZPE between diamond and graphite was estimated in ref. 14 to be in the range of $0.77-1.15 \mathrm{~kJ} \mathrm{~mol}^{-1}$ in favor of graphite. This reduces the difference of their electronic energies to the range of $0.14-0.78 \mathrm{~kJ} \mathrm{~mol}^{-1}$ still in favor of graphite.

\section{B. Hybridization (in)dependence of one-center energies for group IV atoms with nonpolar bonds}

B.1 Core attraction hybridization (in)dependence. The core attraction energy of an atom depends on the populations $q_{m}$ of the respective hybrids and the core-attraction matrix elements for the $m$-th hybrid, ${ }^{31}$ namely $U_{\mathrm{s}} s_{m}{ }^{2}+U_{\mathrm{p}}\left(1-s_{m}{ }^{2}\right)$, where $U_{\mathrm{s}}$ and $U_{\mathrm{p}}$ are the core-attraction parameters for s- and p-AOs; $s_{m}$ is the amplitude of the s-AO in the $m$-th hybrid:

$$
\sum_{m=1}^{4} q_{m}\left[U_{\mathrm{s}} s_{m}^{2}+U_{\mathrm{p}}\left(1-{s_{m}}^{2}\right)\right]
$$

The weights of the s-AOs in the system of hybrids are subject to the normalization condition:

$$
\sum_{m=1}^{4} s_{m}^{2}=1
$$

Inserting $q_{m}=1$ (no polar bonds) and using eqn (B1), we get the constant one-center attraction: $U_{\mathrm{s}}+3 U_{\mathrm{p}}$. Thus, in group IV atoms with nonpolar bonds, the one-center attraction energy does not depend on hybridization.

B.2 One-center Coulomb interactions. The one-center interhybrid Coulomb interactions are expressed as a sum of the products of populations of the hybrids times the Coulomb matrix elements $g_{k m}(k \neq m)$ between hybrids, which are known: ${ }^{31}$

$$
g_{k m}=C_{4}+C_{5}\left[s_{m}^{2}+s_{k}^{2}\right]+C_{3} s_{m}^{2} s_{k}^{2}
$$

Here, $C_{i}$ are, as well, known combinations of the atomic Slater-Condon parameters ${ }^{31}$ whose explicit forms are not required. Inserting the populations of the hybrids $q_{m}=1$ and performing the summation with the use of the normalization condition eqn (B1), we get:

$$
\frac{1}{4} \sum_{m} \sum_{k \neq m} q_{k} q_{m} g_{k m}=3 C_{4}+\frac{3}{2} C_{5}+C_{3}-\frac{C_{3}}{4} \sum_{m} s_{m}{ }^{4} .
$$

Now, we complement the Coulomb interaction between different hybrids in eqn (B2) with the interaction of electrons within hybrids:

$$
\frac{1}{4} \sum_{m} q_{m}^{2} g_{m}
$$

The intrahybrid Coulomb matrix elements $g_{m}$ are, as well, known: ${ }^{31}$

$$
g_{m}=C_{1}+C_{2} s_{m}{ }^{2}+C_{3} s_{m}{ }^{4}
$$

Inserting $q_{m}=1$, performing summation and using again the normalization condition from eqn (B1), we see that the terms quadratic in $s_{m}$ sum up to a constant $C_{2} / 4$, whereas the sum of terms quartic in $s_{m}$ exactly cancels the corresponding term in the interhybrid interaction energy in eqn (B2). Thus, we arrive at the main conclusion of this Appendix: the energy of a group IV atom forming no polar bonds is hybridization-independent. 


\section{Parameter extraction by LOBSTER}

In order to clarify the key parameters of this study, the resonance integrals for carbon and silicon atoms, we first performed DFT calculations by employing the Vienna Ab initio Simulation Package (VASP) ${ }^{62-65}$ using the PBE exchange-correlation functional ${ }^{66}$ and PAW pseudo-potentials. ${ }^{67,68}$ A convergence criterion of at least $10^{-5} \mathrm{eV}$ was used, and the atomic positions were optimized until the Hellmann-Feynman forces fell below $5 \times 10^{-3} \mathrm{eV} \mathrm{\AA}^{-1}$.

We then utilized the LOBSTER package to project the DFT results onto a local basis of contracted Slater-type orbitals using primitive unit cells. ${ }^{43-45}$ The processing yields the values of the resonance integrals between the atomic orbitals at the interatomic separations available in the respective crystals (Fig. 6 and 7).

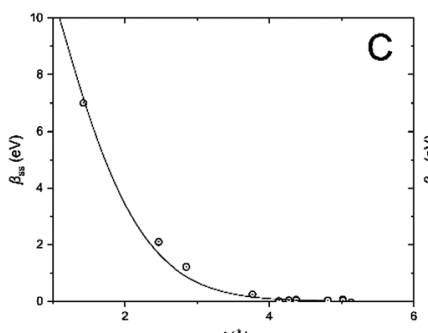

$d(A)$

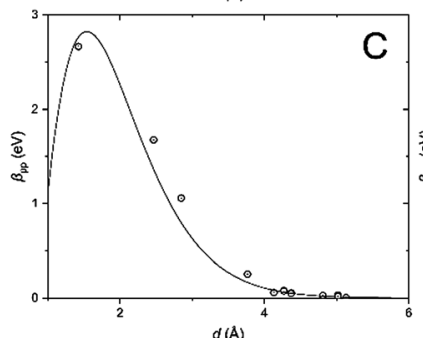

$d(\mathrm{~A})$

Fig. 6 Distance dependence of the resonance integrals for carbon as obtained by LOBSTER and fitted as being proportional to the overlap integrals.
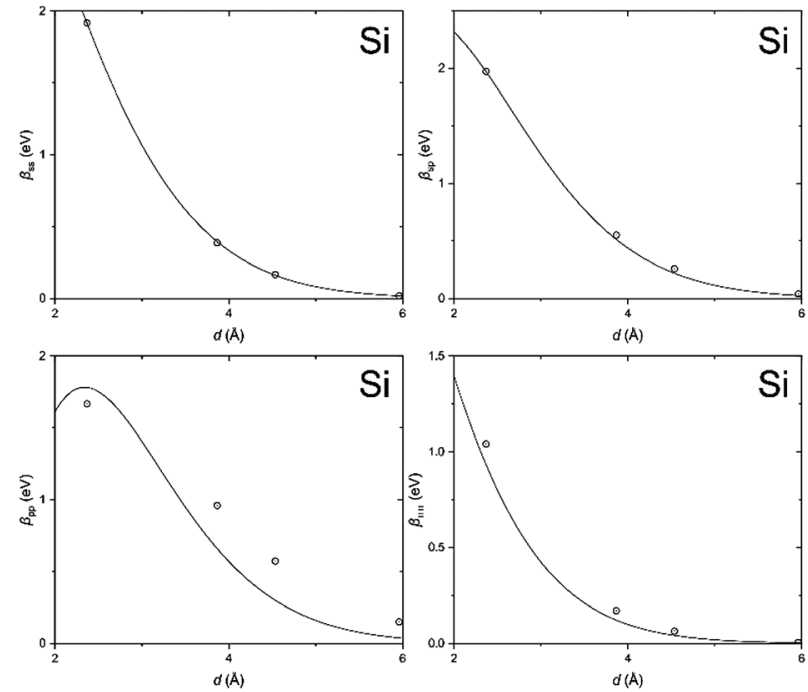

Fig. 7 Same as Fig. 6, but for silicon.
Table 2 Parameters $\beta_{\mu \nu}^{0}(\mathrm{eV})$ extracted from the LOBSTER projection of our DFT calculation on graphite and their values in the standard ${ }^{69}$ and "local"47 MNDO parametrizations

\begin{tabular}{|c|c|c|c|c|}
\hline \multirow[b]{2}{*}{$\mu \nu$} & \multicolumn{2}{|l|}{$\mathrm{C}$} & \multicolumn{2}{|l|}{$\mathrm{Si}$} \\
\hline & Extracted & $\mathrm{MNDO}^{47}$ & Extracted & MNDO $^{69}$ \\
\hline SS & 16.506 & 17.133 & 5.104 & 4.256 \\
\hline $\mathrm{sp}$ & 12.280 & 13.238 & 4.835 & \\
\hline $\mathrm{pp}$ & 8.488 & 9.343 & 4.624 & \\
\hline$\pi \pi$ & 8.437 & 10.306 & 4.201 & \\
\hline
\end{tabular}

The DFT-derived hopping matrix elements were fitted as products of the energy scales $\beta_{\mu \nu}^{0}$ and the overlap integrals $S_{\mu \nu}$ of the Slater-type orbitals (Table 2) with the orbital exponents taken from the standard MNDO parametrization. ${ }^{48,69}$ The results of the numerical fit reproduce characteristic features of the MNDO system of parameters. For carbon, there is a strong dependence of the scaling factors on the orbital type (a factor of about two between the ss and pp energy scales) as well as the MNDO specific combination rule: $2 \beta_{\mathrm{sp}}^{0}=\beta_{\mathrm{ss}}^{0}+\beta_{\mathrm{pp}}^{0}$; for silicon, by contrast, there is only a weak dependence of the scales on the orbital type.

\section{Acknowledgements}

This work is supported by the Volkswagenstiftung (grant no. 151110) "Deductive Quantum Molecular Mechanics of Carbon Allotropes" in the frame of a trilateral project between scholars and scientists from the Ukraine, Russia, and Germany. The authors are thankful to Prof. R. Hoffmann (Cornell) for valuable discussion on lonsdaleite and to Prof. Dr W. H. E. Schwarz (Siegen, Bejing) for discussion on the importance of repulsion terms. Dr A. Tokmachev (Moscow) is acknowledged for drawing ALT's attention to the problem of corrugation in silicene. The authors are also thankful to the Referees for valuable comments, which helped to improve the final version of this paper.

\section{Notes and references}

1 SACADA Database http://sacada.sctms.ru/.

2 R. Hoffmann, A. A. Kabanov, A. A. Golov and D. M. Proserpio, Angew. Chem., Int. Ed., 2016, 55, 10962-10976.

3 M. Esser, A. A. Esser, D. M. Proserpio and R. Dronskowski, Carbon, 2017, 121, 154-162.

4 M. Esser and R. Dronskowski, Carbon, 2017, 123, 708-716.

5 E. P. Randviir, D. A. C. Brownson and C. E. Banks, Mater. Today, 2014, 17, 426-432.

6 S. F. A. Acquaha, A. V. Penkova, D. A. Markelov, A. S. Semisalova, B. E. Leonhardt and J. M. Magi, ECS J. Solid State Sci. Technol., 2017, 6, M3155-M3162.

7 W. Jun Lee, U. Narayan Maiti, J. Min Lee, J. Lim, T. H. Han and S. O. Kim, Chem. Commun., 2014, 50, 6818.

8 T. Hom, W. Kiszenikand and B. Post, J. Appl. Crystallogr., 1975, 8, 457-458.

9 P. Trucano and R. Cheb, Nature, 1975, 258, 136-137.

10 F. P. Bundy and J. S. Kasper, J. Chem. Phys., 1967, 46, 3437-3446. 
11 X.-L. Sheng, Q.-B. Yan, F. Ye, Q.-R. Zheng and G. Su, Phys. Rev. Lett., 2011, 106, 155703; and reference therein.

12 H. W. Kroto, J. R. Heath, S. C. Obrien, R. F. Curl and R. E. Smaliey, Nature, 1985, 318, 162; S. Iijima, Nature, 1991, 354, 56; Y. Liu, G. Wang, Q. Huang, L. Guo and X. Chen, Phys. Rev. Lett., 2012, 108, 225505.

13 I. A. Baburin, D. M. Proserpio, V. A. Saleev and A. V. Shipilova, Phys. Chem. Chem. Phys., 2015, 17, 1332-1338.

14 W. Grochala, Angew. Chem., Int. Ed., 2014, 53, 3680-3683.

15 H. W. Day, Am. Mineral., 2012, 97, 52-62.

16 R. J. Corruccini and J. J. Gniewek, Natl. Bur. Stand., Monogr., 1960, 21.

17 J.-W. Jiang, B.-S. Wang, J.-S. Wang and H. S. Park, J. Phys.: Condens. Matter, 2015, 27, 083001.

18 Picture by J. Hedberg licensed under a Creative Commons Attribution-NonCommercial-ShareAlike 3.0 Unported License, http://www.jameshedberg.com/scienceGraphics. php?sort=all\&id=graphene-sheet-atoms.

19 V. V. Tarasov, DAN SSSR, 1945, 46, 22; V. V. Tarasov, Zh. Fiz. Khim., 1950, 24, 111.

20 W. DeSorbo and W. W. Tyler, J. Chem. Phys., 1953, 21, 1660-1663; E. Pop, V. Varshney and A. K. Roy, MRS Bull, 2012, 37, 1273.

21 W. Wang, S. Dai, X. Li, J. Yang, D. J. Srolovitz and Q. Zheng, Nat. Commun., 2015, 6, 7853.

22 N. L. Allinger and U. Burkert, Molecular Mechanics, An American Chemical Society Publication, 1982.

23 A. Leach, Molecular Modelling: Principles and Applications, 2nd edn, Prentice Hall, Harlow, 2001.

24 The 'quantum' MM schemes ${ }^{25-27}$ represent, in fact, tedious interpolations of the multiple energy contributions while for the quantum mechanical quantities, some ad hoc expressions are used.

25 J. Che, T. Çağıın and W. A. Goddard III, Theor. Chem. Acc., 1999, 102, 346.

26 D. W. Brenner, Phys. Rev. B: Condens. Matter Mater. Phys., 1990, 42, 9458.

27 V. G. S. Box, J. Mol. Model., 1997, 3, 124-141.

28 R. McWeeny and B. T. Sutcliffe, Methods of molecular quantum mechanics, AP, London, 1969; R. McWeeny, Methods of Molecular Quantum Mechanics, AP, London, 1992.

29 A. L. Tchougréeff, J. Struct. Chem., 2007, 48, S39-S62 [in Russian]; A. L. Tchougréeff, J. Struct. Chem., 2007, 48, S32-S54 [in English].

30 A. L. Tchougréeff, Hybrid Methods of Molecular Modeling, Springer, 2008.

31 A. L. Tchougréeff, THEOCHEM, 2003, 630, 243-263; A. L. Tchougréeff and A. M. Tokmachev, Int. J. Quantum Chem., 2004, 96, 175-184.

32 A. L. Tchougréeff and R. Dronskowski, Mol. Phys., 2016, 1423-1444.

33 A. L. Tchougréeff, AIP Conf. Proc., 2017, 1906, UNSP 030004; A. L. Tchougréeff, Theor. Chem. Acc., 2018, 137, 138.

34 It is not always realized but the hardness - rigidity - of a body or material is a consequence not of "bonding" contributions because these are largely concave functions of inter-atomic separations, providing negative (i.e., softening) contribution to the elastic moduli. Rather, the core repulsion terms preventing nuclei to come too close together and therefore produce large convex contribution (positive second derivative). Thus, one should not be surprised that diamond with a larger coefficient at the repulsion term in the total energy is harder than graphite.

35 J. Pople and D. Beveridge, Approximate Molecular Orbital Theory, McGraw-Hill, 1970; I. Levine, Quantum Chemistry, Prentice Hall, 4th edn, 1991; C. J. Cramer, Essentials of Computational Chemistry, Wiley, Chichester, 2002; A. Szabo, N. S. Oslund, Modern Quantum Chemistry: Introduction to Advanced Electronic Structure Theory, Dover, 1996.

36 A. L. Tchougréeff and A. M. Tokmachev, Int. J. Quantum Chem., 2006, 106, 571-587.

37 A. M. Tokmachev and A. L. Tchougréeff, J. Comput. Chem., 2001, 22, 752-764.

38 A. M. Tokmachev and A. L. Tchougréeff, Int. J. Quantum Chem., 2002, 88, 403-413.

39 A. M. Tokmachev and A. L. Tchougréeff, J. Comput. Chem., 2005, 26, 491-505.

40 P. R. Wallace, Phys. Rev., 1947, 71, 622.

41 C. A. Coulson and H. C. Longuet-Higgins, Proc. Roy. Soc. Lond. A, 1947, 191, 16-32; C. A. Coulson and A. Golebiewski, Proc. Phys. Soc., 1961, 78, 1310.

42 G. C. Abell, Phys. Rev. B: Condens. Matter Mater. Phys., 1985, 31, 6184-6196.

43 V. L. Deringer, A. L. Tchougréeff and R. Dronskowski, J. Phys. Chem. A, 2011, 115, 5461-5466.

44 S. Maintz, V. L. Deringer, A. L. Tchougréeff and R. Dronskowski, J. Comput. Chem., 2013, 34, 2557-2567.

45 S. Maintz, V. L. Deringer, A. L. Tchougréeff and R. Dronskowski, J. Comput. Chem., 2016, 37, 1030-1035.

46 A. M. Tokmachev and A. L. Tchougréeff, J. Phys. Chem. A, 2005, 109, 7613-7620.

47 A. L. Tchougréeff, A. M. Tokmachev and R. Dronskowski, J. Phys. Chem. A, 2009, 113, 11406-11415.

48 M. J. S. Dewar and W. Thiel, J. Am. Chem. Soc., 1977, 99, 4899-4907.

49 R. A. Andrievski, Int. J. Refract. Met. Hard Mater., 2001, 19, 447.

50 F. P. Bundy and J. S. Kasper, J. Chem. Phys., 1967, 46, 3437-3446.

51 C. Kittel, Introduction to Solid State Physics, Wiley, 1996.

52 It is well known that the MNDO parameterization usually underestimates the Si-Si bond lengths. For example, in the original work $^{69}$ the calculated value of the $\mathrm{Si}-\mathrm{Si}$ bond length in $\mathrm{H}_{3} \mathrm{Si}-\mathrm{SiH}_{3}$ equals $2.284 \AA$, while the experimental value is 2.352 A. It is also discussed in R. Hull, Properties of Crystalline Silicon, IET, 1999 that both MNDO parameterizations for silicon fail to reproduce the experimental structure of silicon correctly.

53 A. D. Elliott, Acta Crystallogr., Sect. B: Struct. Sci., 2010, 66, 271-279.

54 R. H. Wentorf and J. S. Kasper, Science, 1963, 338-339. 
55 K. Takeda and K. Shiraishi, Phys. Rev. B: Condens. Matter Mater. Phys., 1994, 50, 14916.

56 B. Haberl, T. A. Strobel and J. E. Bradby, Appl. Phys. Rev., 2016, 3, 040808.

57 L. C. Lew Yan Voon, J. Zhu and U. Schwingenschlögl, Appl. Phys. Rev., 2016, 3, 040802; E. Durgun, S. Tongay and S. Ciraci, Phys. Rev. B: Condens. Matter Mater. Phys., 2005, 72, 075420.

58 A. Tchougréeff, Abstracts ACS, 2017, 254, 209.

59 A. Zunger, Phys. Rev. B: Condens. Matter Mater. Phys., 1978, 17, 626-642.

60 Knovel database, http://why.knovel.com/accessed Oct 2013.

61 NIST database, https:/webbook.nist.gov/cgi/formula?ID= C7782425\&Mask=2.

62 G. Kresse and J. Hafner, Phys. Rev. B: Condens. Matter Mater. Phys., 1993, 47, 558-561.

63 G. Kresse and J. Hafner, Phys. Rev. B: Condens. Matter Mater. Phys., 1994, 49, 14251-14269.

64 G. Kresse and J. Furthmüller, Phys. Rev. B: Condens. Matter Mater. Phys., 1996, 54, 11169-11186.
65 G. Kresse and J. Furthmüller, Comput. Mater. Sci., 1996, 6, 15-50.

66 J. P. Perdew, K. Burke and M. Ernzerhof, Phys. Rev. Lett., 1996, 77, 3865-3868.

67 P. E. Blöchl, Phys. Rev. B: Condens. Matter Mater. Phys., 1994, 50, 17953-17979.

68 G. Kresse and D. Joubert, Phys. Rev. B: Condens. Matter Mater. Phys., 1999, 59, 1758-1775.

69 M. J. S. Dewar, M. L. McKee and H. S. Rzepa, J. Am. Chem. Soc., 1978, 100, 3607.

70 P. Németh, L. A. J. Garvie, T. Aoki, N. Dubrovinskaia, L. Dubrovinsky and R. Buseck, Nat. Commun., 2014, 5, 5447.

71 Z. Pan, H. Sun, Y. Zhang and C. Chen, Phys. Rev. Lett., 2009, 102, 055503.

72 L. Quingkun, S. Yi, L. Zhiyuan and Z. Yu, Scr. Mater., 2011, 65, 229-232.

73 T. Gruber, K. Liao, T. Tsatsoulis, F. Hummel and A. Grüneis, Phys. Rev. X, 2018, 8, 021043. 